\title{
MUTUAL APOSYNDESIS
}

\section{L. HAGOPIAN ${ }^{1}$}

1. Introduction. Let $M$ be a continuum (a connected topological space) and let $x$ and $y$ be distinct points of $M$. If $M$ contains a continuum $H$ and an open subset $U$ such that $x \in U \subset H \subset M-\{y\}$, then $M$ is said to be aposyndetic at $x$ with respect to $y^{2}$. This familiar property can be strengthened as follows. If $M$ contains two disjoint continua $H$ and $K$ such that $x$ is contained in the interior of $H$ and $y$ is contained in the interior of $K$, then $M$ is said to be mutually aposyndetic at $x$ and $y$. This paper is concerned with the relationship between these two properties. Certain connected topological product spaces are shown to be mutually aposyndetic. Cutting properties in nonmutually aposyndetic, compact, metric continua are established. Strict nonmutual aposyndesis is defined. This property is related to mutual aposyndesis as indecomposability is related to aposyndesis. Just as indecomposability in compact metric continua can be characterized in terms of cutting properties, so can strict nonmutual aposyndesis. Quasi-composants are introduced. It is shown that the theory of composants in indecomposable continua is valid for quasicomposants in certain strictly nonmutually aposyndetic continua. Indecomposability in a chainable continuum is characterized in terms of strict nonmutual aposyndesis in a product space. This result is used to verify that a certain aposyndetic, homogeneous continuum in Euclidean 3-space is strictly nonmutually aposyndetic.

Definitions. If a continuum $M$ is mutually aposyndetic at every pair of distinct points, then $M$ is said to be mutually aposyndetic. If for a point $p$ in $M$ there exists a point $q$ in $M-\{p\}$ such that $M$ is not mutually aposyndetic at $p$ and $q$, then $M$ is said to be nonmutually aposyndetic at $p$. If $M$ is nonmutually aposyndetic at each of its points, then $M$ is said to be totally nonmutually aposyndetic. A continuum $M$ is said to be strictly nonmutually aposyndetic if each pair of continua in $M$ which have interiors intersect. A continuum $M$ is locally remotely connected at a point $p$ in $M$ if for each open set $U$ in $M$ containing $p$ there exists an open set $V$ in $U$ containing $p$, such that $M-V$ is connected. A continuum $M$ is said to be locally remotely connected if for each point $p$ in $M, M$ is locally remotely connected at $p$.

Received by the editors January 8, 1969.

1 Supported by the Air Force Office of Scientific Research.

${ }^{2}$ For definitions of unfamiliar terms and phrases, see [4] and [5]. 
2. Mutual aposyndesis. F. Burton Jones has shown that the topological product of two nondegenerate continua is aposyndetic [4, Theorem 7]. Results at the end of this paper indicate that product spaces are not always mutually aposyndetic. However, some product spaces do have this property.

THEOREM 1. The topological product of a nondegenerate aposyndetic continuum $H_{1}$ with a nondegenerate aposyndetic continuum $\mathrm{H}_{2}$ is mutually aposyndetic.

Proof. Let $\left(x_{1}, x_{2}\right)$ and $\left(y_{1}, y_{2}\right)$ be two distinct points in $H_{1} \times H_{2}$. Assume without loss of generality that $x_{1}$ and $y_{1}$ are distinct points in $H_{1}$. Since $H_{1}$ is aposyndetic, a continuum $C$ and an open set $Q$ exist in $H_{1}$ such that $x_{1}$ is contained in the interior of $C, y_{1}$ is contained in $Q$, and $C \subset H_{1}-\mathrm{Cl}(Q),(\mathrm{Cl}(Q)$ is the closure of $Q)$. Let $z$ be a point of $H_{2}$ distinct from $x_{2}$. Since $H_{2}$ is aposyndetic, a continuum $K$ exists in $H_{2}-\{z\}$ such that $x_{2}$ is in the interior of $K$. Define $L=C \times K, U$ $=\mathrm{Cl}(Q) \times H_{2}$, and $V=\left[\mathrm{Cl}(Q) \times H_{2}\right] \cup\left[H_{1} \times\{z\}\right]$. One can see that $L$ and $V$ are disjoint continua with $\left(x_{1}, x_{2}\right)$ in the interior of $L$ and $\left(y_{1}, y_{2}\right)$ in the interior of $V$. It follows that $H_{1} \times H_{2}$ is mutually aposyndetic.

EXAmple 1. If the continua $H_{1}$ and $H_{2}$ are not both aposyndetic, then $H_{1} \times H_{2}$ may fail to be mutually aposyndetic. To see this, define $H_{1}$ to be the plane continuum consisting of the join of the closure of $\{(0,1 / n) \mid n=1,2,3, \cdots\}$ with the point $(1,0)$ and the join of the closure of $\{(1,-1 / n) \mid n=1,2,3, \cdots\}$ with the point $(0,0)$. Let $H_{2}$ be the unit interval on the $z$-axis. $H_{1} \times H_{2}$ is not mutually aposyndetic at $(0,0,0)$ and $(1,0,0)$.

THEOREM 2. The topological product of three nondegenerate Hausdorff continua $H_{1}, H_{2}$, and $H_{3}$ is mutually aposyndetic.

Proof. Let $\left(x_{1}, x_{2}, x_{3}\right)$ and $\left(y_{1}, y_{2}, y_{3}\right)$ be two distinct points in $H_{1} \times H_{2} \times H_{3}$. Assume without loss of generality that $x_{1}$ and $y_{1}$ are distinct points in $H_{1}$. Since the product of two nondegenerate continua is aposyndetic, a continuum $K$ and an open set $G$ exist in $H_{1} \times H_{2}$ such that $\left(x_{1}, x_{2}\right)$ is in the interior of $K,\left(y_{1}, y_{2}\right)$ is in $G, K C\left(H_{1} \times H_{2}\right)-\mathrm{Cl}(G)$ and $x_{1}$ is not in $P_{1} \mathrm{Cl}(G)$ (the projection of $\mathrm{Cl}(G)$ in to $H_{1}$ ). Let $U$ be an open set and $z$ be a point in $H_{3}$ such that $x_{3}$ is in $U$ and $z$ is in $H_{3}$ $-\mathrm{Cl}(U)$. Define the set

$$
L=\left\{(p, q, r) \in H_{1} \times H_{2} \times H_{3} \mid(p, q) \in K \text { and } r \in \mathrm{Cl}(U)\right\} .
$$

Let $H_{3}\left(x_{1}, x_{2}\right)$ denote the set of all points $(p, q, r)$ of $H_{1} \times H_{2} \times H_{3}$ such that $p=x_{1}$ and $q=x_{2}$. Let $C=H_{3}\left(x_{1}, x_{2}\right) \cup L$. Note that $C$ is a contin- 
uum in $H_{1} \times H_{2} \times H_{3}$ which contains $\left(x_{1}, x_{2}, x_{3}\right)$ in its interior. Now define the sets

$$
R=\left\{(p, q, r) \in H_{1} \times H_{2} \times H_{3} \mid(p, q) \in \mathrm{Cl}(G)\right\}
$$

and

$$
T=\left\{(p, q, r) \in H_{1} \times H_{2} \times H_{3} \mid p \in P_{1} \mathrm{Cl}(G), q \in H_{2} \text {, and } r=2\right\} .
$$

Let $w$ be a point of $H_{2}$ distinct from $x_{2}$. Let $H_{1}(w, z)$ denote the set of all points $(p, q, r)$ of $H_{1} \times H_{2} \times H_{3}$ such that $q=w$ and $r=z$. Define $D=H_{1}(w, z) \cup R \cup T$. The set $D$ is a continuum which misses $C$ and contains the point $\left(y_{1}, y_{2}, y_{3}\right)$ in its interior. Therefore $H_{1} \times H_{2} \times H_{3}$ is mutually aposyndetic at $\left(x_{1}, x_{2}, x_{3}\right)$ and $\left(y_{1}, y_{2}, y_{3}\right)$. It follows that $H_{1} \times H_{2} \times H_{3}$ is mutually aposyndetic.

Theorem 3. Suppose that $x_{1}, x_{2}, \cdots$ and $y_{1}, y_{2}, \cdots$ are sequences in a continuum $M$ such that

(1) for each positive integer $i, x_{i}$ is a point of $M$ and $y_{i}$ is a point of $M-\left\{x_{i}\right\}$

(2) $x_{1}, x_{2}, \cdots$ converges to a point $x$ and $y_{1}, y_{2}, \cdots$ converges to a point $y$ distinct from $x$; and

(3) for each $i, M$ is not mutually aposyndetic at $x_{i}$ and $y_{i}$.

Then $M$ is not mutually aposyndetic at $x$ and $y$.

Proof. Assume that $M$ is mutually aposyndetic at $x$ and $y$. It follows that two mutually disjoint subcontinua $H$ and $K$ of $M$ exist, with $x$ and $y$ contained in $\operatorname{int}(H)(\operatorname{int}(H)$ is the interior of $H)$ and $\operatorname{int}(K)$, respectively. Since the sequence $x_{1}, x_{2}, \cdots$ converges to $x$, and $y_{1}, y_{2}, \cdots$ converges to $y$, there is a positive integer $i$ such that $x_{i}$ is in $\operatorname{int}(H)$ and $y_{i}$ is in int $(K)$. But this contradicts the fact that $M$ is not mutually aposyndetic at $x_{i}$ and $y_{i}$. Therefore $M$ is not mutually aposyndetic at $x$ and $y$.

THEOREM 4. If $M$ is a compact metric continuum, the set $N$ of points of $M$ at which $M$ is nonmutually aposyndetic is the union of a countable number of closed sets (i.e., $N$ is an $F_{\sigma}$ set).

Proof. Let $d$ be a metric on $M$. For each positive integer $n$, define $M_{n}$ to be the set of all points $p$ in $M$ such that for some point $q$ of $M$, the continuum $M$ is not mutually aposyndetic at $p$ and $q$, and $d(p, q) \geqq 1 / n$. Let $n$ be a natural number, and let $x$ be in $\operatorname{Cl}\left(M_{n}\right)$. It follows that there are two sequences $x_{1}, x_{2}, \ldots$ and $y_{1}, y_{2}, \ldots$ in $M_{n}$ such that

(1) for each positive integer $i, M$ is not mutually aposyndetic at $x_{i}$ and $y_{i}$, and $d\left(x_{i}, y_{i}\right) \geqq 1 / n$; 
(2) $x_{1}, x_{2}, \cdots$ converges to $x$; and

(3) $y_{1}, y_{2}, \ldots$ converges to a point $y$ in $M$.

By Theorem 3, $M$ is not mutually aposyndetic at $x$ and $y$. Furthermore $d(x, y) \geqq 1 / n$. Therefore, $x$ is in $M_{n}$ and $M_{n}$ is closed. Clearly $\mathrm{U}_{n} M_{n}$ is the set of points at which $M$ is nonmutually aposyndetic.

Theorems 3 and 4 remain true if the words "nonmutual aposyndesis" are everywhere replaced by "nonaposyndesis" [4, Theorems 1 and 4]. If $y$ is a point of a compact continuum $M$, and $L$ is the set of points $x$ of $M-\{y\}$ such that $M$ is not aposyndetic at $x$ with respect to $y$, then $L \cup\{y\}$ is a continuum [4, Theorem 3]. Let $J$ be the set which consists of all points $x$ of $M-\{y\}$ such that $M$ is not mutually aposyndetic at $x$ and $y$. The set $J \cup\{y\}$ is closed but not necessarily connected.

3. $C$-cut points. If a compact metric continuum $M$ is totally nonaposyndetic, then $M$ must contain a weak cut point [4, Theorem 12]. Example 2 (at the end of this paper) indicates that even the strongest form of nonmutual aposyndesis (strict nonmutual aposyndesis) does not insure the existence of a weak cut point. However, a more general type of cutting is present in totally nonmutual aposyndetic continua.

Definition. If $p, r$, and $s$ are distinct points in a continuum $M$ and for each subcontinuum $K$ of $M$ containing $p$ in its interior, every subcontinuum of $M$ containing $r$ and $s$ meets $K$, then $p$ is a $C$-cut point in $M$ and is said to $C$-cut $M$ between $r$ and $s$.

THEOREM 5. If a compact metric continuum $M$ contains an open set $K$ and a set $L$ such that for each point $x$ in $K$, there exists a point $y$ in $L-\{x\}$ such that $M$ is not mutually aposyndetic at $x$ and $y$, then given a point $r$ in $M-L$, there exists a point $s$ in $K$ such that if $p$ is a point in $L$ and $M$ is not mutually aposyndetic at $p$ and $s$, then $p C$-cuts $M$ between $r$ and $s$.

Proof. This theorem follows directly from a general theorem due to E. E. Grace [3, Theorem 4]. The open set $D$ in [3] corresponds to the set $K$. The collection $E$ in [3] can be taken to consist of all finite subcollections of open sets from a countable base and the collection $G$ can be taken to consist of all continua in $M-\{r\}$ which have a point of $L$ in their interior. The point $F(g)$ in [3] would be the point $r$ for each $g$ in $G$.

Corollary 1. If a compact metric continuum $M$ contains a point $p$ and an open set $K$ such that for each point $x$ in $K, M$ is not mutually aposyndetic at $p$ and $x$, then given a point $r$ in $M-\{p\}$, there exists $a$ point $s$ in $K$ such that $p C$-cuts $M$ between $r$ and $s$. 
CoROllary 2. If a compact metric continuum $M$ is totally nonmutually aposyndetic, then $M$ contains a $C$-cut point.

Proof. Let $r$ be a point in $M$. Define

$R=\{x \in M-\{r\} \mid M$ is not mutually aposyndetic at $x$ and $r\}$.

If $R$ is somewhere dense in $M$, then by Corollary 1 and Theorem 3 the point $r C$-cuts $M$ between some two points. If $R$ is nowhere dense in $M$, then the conclusion follows from Theorem 5 . The open set $K$ in Theorem 5 would be contained in $M-R$ and the set $L$ would be $M-\{r\}$.

4. Strict nonmutual aposyndesis. Strictly nonmutually aposyndetic compact metric continua can be characterized in terms of this cutting property.

Theorem 6. A compact metric continuum $M$ is strictly nonmutually aposyndetic if and only if for each open set $G$ and each point $p$ in $M$, there exist two points $r$ and $s$ in $G$ such that $p C$-cuts $M$ between $r$ and $s$.

Proof. Necessity follows immediately from Corollary 1 . To see that this condition is sufficient, assume that there exist distinct points $p$ and $q$ in $M$ such that $M$ is mutually aposyndetic at $p$ and $q$. By definition there exist two disjoint continua $H$ and $K$ in $M$ such that $p$ is in the interior of $H$ and $q$ is in the interior of $K$. But then $p$ does not $C$-cut $M$ between any two points in the interior of $K$. It follows that if $M$ has the stated cutting property then $M$ must be strictly nonmutually aposyndetic.

THEOREM 7. If an aposyndetic continuum $M$ is strictly nonmutually aposyndetic, then $M$ does not contain a weak cut point and is locally remotely connected.

Proof. Given three distinct points $x, y$, and $z$ in $M$, since $M$ is aposyndetic, a continuum $H$ and an open set $U$ exist such that $x \in U$ $\subset H \subset M-\{y\}$, and a continuum $K$ and an open set $V$ exist such that $z \in V \subset K \subset M-\{y\}$. Because $M$ is strictly nonmutually aposyndetic, $H$ and $K$ intersect. It follows that $H \cup K$ is a continuum which contains $x$ and $z$ and misses $y$. Therefore $y$ does not cut $M$ weakly between $x$ and $z$.

To see that $M$ is locally remotely connected, let $p$ be a point in $M$ and let $G$ be an open set containing $p . M$ is semi-locally-connected $[4$, Theorem 0$]$. There exists an open set $U$ in $G$ containing $p$ such that $M-U$ has a finite number of components. Since the point $p$ is not a weak cut point, the components of $M-U$ can be joined together 
by a finite number of continua in $M-\{p\}$. Let $L$ denote the sum of these continua. The set $(M-U) \cup L$ is a continuum in $M-\{p\}$ which contains $M-U$. It follows that $M$ is locally remotely connected at $p$.

Definition. If $p$ is a point of a continuum $M$, then the $p$-quasicomposant of $M$ consists of $p$ together with the union of all continua $K$, if any, such that $p \in K \subset M-H$ for some continuum $H$ in $M$ which has an interior.

Obviously for each point $p$ in a continuum, the $p$-quasi-composant is contained in the $p$-composant. In a locally connected continuum, quasi-composants are composants. Every quasi-composant of a compact metric continuum $M$ is the sum of countably many subcontinua of $M$. This can be proved by making a slight modification in Moore's proof of the theorem which states that every composant of a compact metric continuum is the sum of countably many proper subcontinua [5, Theorem 136, p. 58]. If in this proof $L$ is defined to be the $A$-quasi-composant of $M$ (instead of the $A$-composant of $M$ ) and $G$ is defined to be the countable set consisting of all finite subcollections of open sets from a countable base which cover interior containing subcontinua of $M$, then it is easily seen that the set $T$ in Moore's proof which is the union of countably many subcontinua of $M$ is identical with $L$. Using a category argument, one can show that if a compact metric continuum $M$ is strictly nonmutually aposyndetic, then $M$ has uncountably many quasi-composants.

THEOREM 8. If $M$ is the topological product of two continua $H_{1}$ and $\mathrm{H}_{2}$, then each quasi-composant of $M$ is dense in $M$.

Proof. Let $(p, q)$ be a point and let $G$ be a basic open set in the product space $M$. Since the $p$-composant of $H_{1}$ is dense in $H_{1}$, an open set $U$ in $H_{1}$ and a continuum $K$ in $H_{1}$ exist, such that $K$ meets $P_{1}(G)$ (the projection of $G$ into $H_{1}$ ) and $p \in K C H_{1}-\mathrm{Cl}(U)$. The $q$-composant of $\mathrm{H}_{2}$ is dense in $\mathrm{H}_{2}$. A point $r$ in $\mathrm{H}_{2}$ and a continuum $\mathrm{L}$ in $\mathrm{H}_{2}$ exist, such that $q \in L \subset H_{2}-\{r\}$ and $L$ meets $P_{2}(G)$. Let $t$ be a point of $K \cap P_{1}(G)$. Define

$$
A=K \times\{q\}, \quad B=\{t\} \times L, \quad C=H_{1} \times\{r\}, \text { and } D=\mathrm{Cl}(U) \times H_{2} .
$$

$A \cup B$ is a continuum in $M$ which contains $(p, q)$ and meets $G$. The set $C \cup D$ is a continuum in $M$ which has an interior. $A \cup B$ is contained in $M-(C \cup D)$. It follows that each quasi-composant is dense in $M$.

THEOREM 9. If $M$ is the topological product of two chainable indecomposable continua $I_{1}$ and $I_{2}$, and for each point $p$ in $I_{1}$ and each point $q$ in $I_{2}, C_{p q}$ is defined to be the set of all points $(x, y)$ in $M$ such that $x$ is in 
the p-composant of $I_{1}$ and $y$ is in the q-composant of $I_{2}$, then for each point $(p, q)$ in $M$, the $(p, q)$-quasi-composant of $M$ is $C_{p q}$.

Proof. Let $(\mathrm{x}, \mathrm{y})$ be a point of $C_{p q}$. By methods similar to those used in the proof of Theorem 8, one can establish the existence of two disjoint continua $H$ and $K$ in $M$ such that $H$ has an interior and $(p, q)$ and $(x, y)$ are contained in $K$. It follows that $(x, y)$ is in the $(p, q)$-quasi-composant of $M$.

Assume that the point $(x, y)$ is not in $C_{p q}$ and that $(x, y)$ is in the $(p, q)$-quasi-composant of $M$. There exist two disjoint continua $H$ and $K$ in $M$ such that $H$ has an interior and the points $(p, q)$ and $(x, y)$ are in $K$. Chain $I_{1}$ and $I_{2}$ with open sets which are small enough in diameter to insure the following. When the chain of $I_{1}$ is crossed with the chain of $I_{2}$ the resulting "rectangular" array of open sets forms a cover of $M$ with the property that no open set in the array which meets $H$ intersects an open set in the array which meets $K$. The open sets which meet $H$ and the open sets which meet $K$ are two coherent collections. No open set in one collection meets an open set in the other. Assume without loss of generality that $x$ is not in the $p$-composant of $I_{1}$. Since $I_{1}$ is indecomposable, the projection of $K$ onto $I_{1}$ must meet each link of the chain of $I_{1}$. Therefore $K$ must meet every column of the array. Since $H$ has an interior, the projection of $H$ onto $I_{1}$ must meet each link of the chain of $I_{1}$ and the projection of $H$ onto $I_{2}$ must meet each link of the chain of $I_{2}$. Therefore $H$ must meet every column and row of the array. But this is impossible since the open sets which meet $H$ and the open sets which meet $K$ are two coherent collections and the elements of one collection do not meet the elements of the other. It follows that the point $(x, y)$ is not in the $I_{1}(p, q)$-quasi-composant of $M$.

Corollary. If $M$ is the topological product of two chainable indecomposable continua, then $M$ consists of $c$ disjoint quasi-composants.

THeORem 10. If $H$ is a chainable continuum, then $H$ is indecomposable if and only if the topological product $H \times H$ is strictly nonmutually aposyndetic.

Proof. Assume that $H$ is not indecomposable. An open set $U$ and a continuum $K$ exist in $H$ such that $K$ has an interior and $\mathrm{Cl}(U)$ is in $H-K$. Define

$$
A=K \times K \text { and } B=[\mathrm{Cl}(U) \times H] \cup[H \times \mathrm{Cl}(U)] .
$$

$A$ and $B$ are two disjoint continua in $H \times H$ each having interior. It follows that $H \times H$ is not strictly nonmutually aposyndetic. 
If $H \times H$ is not strictly nonmutually aposyndetic, then there exist two disjoint continua $K$ and $L$ in $H \times H$ each having an interior. It follows that the interior of $K$ is contained in one quasi-composant of $H \times H$. By the preceding corollary and Theorem 8 , if $H$ is indecomposable, $H \times H$ consists of $c$ disjoint quasi-composants and each quasi-composant is dense in $H \times H$. Therefore $H$ is decomposable.

ExAmple 2. Let $M$ be the topological product of the pseudo-arc with itself. Since $M$ is the product of two chainable continua, it is embeddable in Euclidean 3-space [1]. The continuum $M$ is aposyndetic [4, Theorem 7]. Since the pseudo-arc is homogeneous, $M$ is homogeneous [2]. Theorem 10 indicates that $M$ is strictly nonmutually aposyndetic. Note that $M$ is also locally remotely connected and does not contain a weak cut point (Theorem 7).

Question. Is the topological product of two indecomposable compact metric continua strictly nonmutually aposyndetic?

\section{BIBLIOGRAPHY}

1. Ralph Bennett, Embedding products of chainable continua, Proc. Amer. Math. Soc. 16 (1965), 1026-1027.

2. R. H. Bing, Concerning hereditarily indecomposable continua, Pacific J. Math. 1 (1951), 43-51.

3. E. E. Grace, Cut sets in totally nonaposyndetic continua, Proc. Amer. Math. Soc. 9 (1958), 98-104.

4. F. B. Jones, Concerning non-aposyndetic continua, Amer. J. Math. 70 (1948), 403-413.

5. R. L. Moore, Foundations of point set theory, Amer. Math. Soc. Colloq. Publ., vol. 13, Amer. Math. Soc., Providence, R. I., 1962.

California Institute of Technology 